\title{
Writing for Change
}

Non peer-reviewed preprint for publication with the Blog of the European Association of Geochemistry November 2020.

\section{By Amy J. V. Riches ${ }^{1,2^{*}}$}

${ }^{1}$ Visiting Honorary Fellow, University of Edinburgh, School of Geoscience, Grant Institute, Edinburgh, EH9 3FE, UK ${ }^{2}$ SETI Institute, 189 Bernardo Ave, Suite 200, Mountain View, CA 94043, United States 


\section{Writing for Change}

"Somewhere, something incredible is waiting to be known" Carl Sagan (1934-1996), Astronomer and Science Communicator. We can ask of this quote, but by who?

We may consider academia as a rousing type of workplace offering freedom to think and conduct research that can promise major new discoveries while educating and training the next generation of decision makers and explorers. An occupation to leap out of bed for each day and thrill in the revelations it can bring - sometimes with more attentive peering down microscopes, wrestling in the laboratory or intellectually, than at other moments. Work that we love, and love to share. As demonstrated by the examples of COVID-19 and the Climate Change Crisis, STEM matters are crucially important to communicate and secure public trust in. Yet, something is not quite right with us and has been so for a very long time. Academia's STEM disciplines represent a sector in which challenges to diversity among permanent staff and institutional leadership are significant and have persisted over many decades; we do not reflect the demographics of the public that we serve. Short insecure contracts account for unhealthy proportions of staff, an employment model impacting and marginalising some minority groups more so than others. In addition to structural barriers, evidence has shown that minority groups are more likely to have negative and harmful workplace experiences also. Further, reports such as the "Research Integrity: a

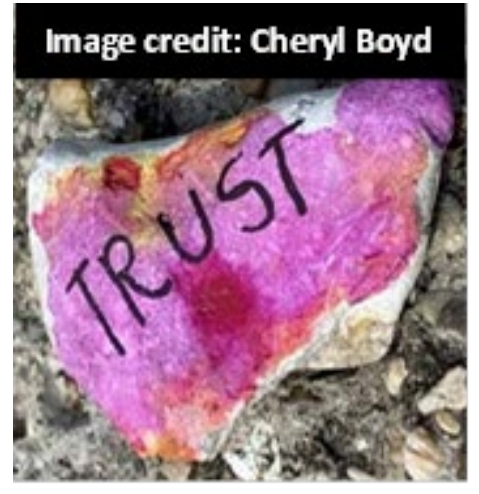

landscape study" by vitae.ac.uk have raised concerns about how this culture of short contracts, as well as over-emphasis on criteria such as metrics, threatens - at systemic levels - compromises to research rigour and reduction in the prevalence of especially challenging study types. Do these combined factors negatively impact STEM attractiveness as well as scientific quality? A lack of diversity is disappointing to face, not least because diverse staff bodies have been shown to be more productive and creative. Hence, diverse mixes are the most capable of exciting, highly-innovative, widely important, and unplanned-for discoveries potentially at quicker pace than otherwise. If we are to optimise inspiration and our scientific efforts then all talent pools must have equitable access and be included at every level.

It is recognised that some actions to address gender equity in STEM are aiding progress, albeit at a snail like pace especially when all forms of opportunity, levels of seniority, and influential leadership positions are considered. But this is nothing in comparison to the well-known and evidenced stagnation in attracting to- and retaining in academic STEM-training and careers people of colour, varying ethnic or religious backgrounds, LGBTQ+ communities, individuals with mental health, physical disabilities, serious health challenges and / or caring responsibilities, and those originating from so-called 'underprivileged' socioeconomic groups. We geochemists, and others in STEM, are highly-trained problem solvers so let's

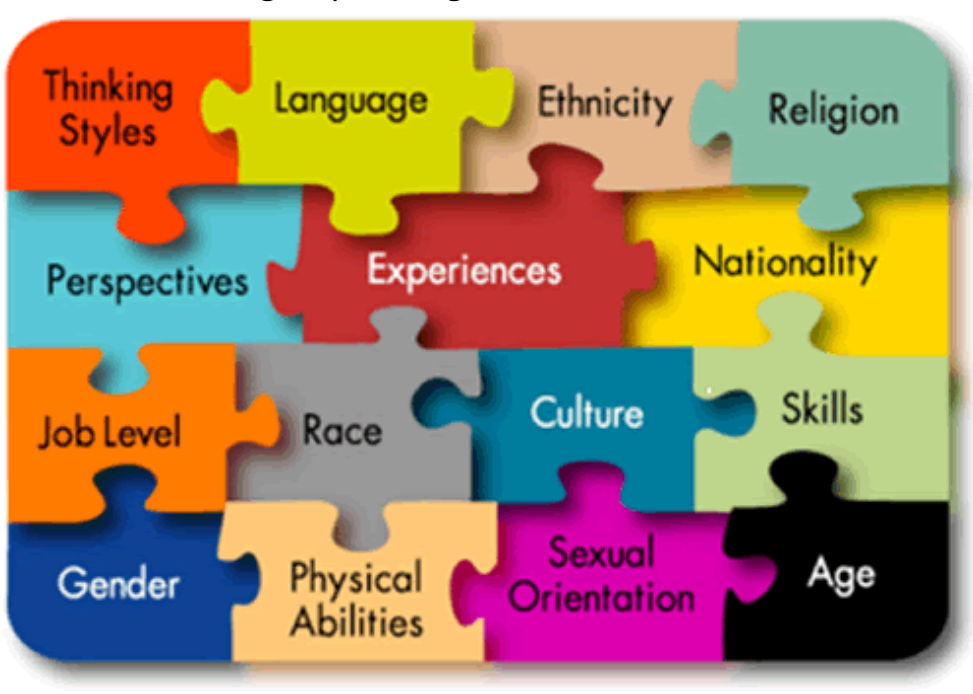
collectively take on this challenge. Why are changes to improve recruitment, retention, and progression so sluggish and, in some cases, underserving or entirely failing scientists of minority groups? Does the academy and STEM have both an ecosystem-model and an image problem? Can these environments and their structures be friendlier to all? What more can and should we do to make STEM subjects, and careers in them, more appealing to- and realistic for those that identify as minorities? Where, in this respect, do our failings begin? 
People and their ideas are the foundation to all science, they are the talent. However complex and challenging the task may be we must not slink from our duties to promote and learn from the good practices that are successful and committed in improving diversity, equity, and friendly inclusion. This should be a recognised and valued part of our careers and contributions. Though long overdue - and we shouldn't wait for the extremes of the unjust deaths of others to act on our principles - the Black Lives Matter movement has brought about positive reforms among the policies and organisational structures of many professional societies of STEM, including the European Association of Geochemistry. Such reforms also help to raise awareness and open discussion of inequalities among the various assessments inherent in academic environments as well as the ways these might be addressed. Based on my personal experiences as a woman and as a witness to our culture, an unavoidable truth that contributes to the broken recruitment and career pipeline of STEM is social conditioning. At what points in the lives of young people do they consider their potential futures? How can the thrill of scientific investigations and discoveries be shared with all kinds of children, and in ways that seize their interest and inspire them for decades ahead?

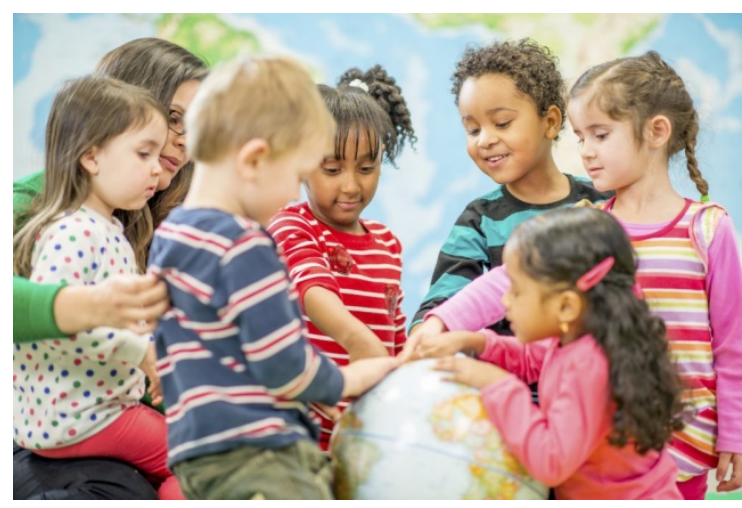

If, as a community, we are loyal to the cause of changing culture so as to champion and improve diversity, equity, and inclusion in academia and STEM - and we accept that social conditioning and aspiration-setting from a young age play a role - then we must collectively dive into fresh ventures that bring the excitement of science to all children in ways to which they can relate. Stirring in children the boldness to dream big while purposefully aiming to uplift those that we currently lack. But how? There are many ways to achieve this goal, where museums, science summer camps, science festivals and other existing outreach activities for schools and wider communities often offer superb experiences that are a vital part of what we do. But not all children have easy access to museums, summer camps / festivals, nor may they be located to regularly gain from STEM-based outreach activities in schools. So what can we do? Are books read and enjoyed in children's own homes the answer? Perhaps this is worth a hearty try as a way to help.

I am from a very humble background, and never imagined I would be the type of person to complete university, hold a doctorate, discover that working on fragments of other worlds is in fact a job, travel to far flung places for work, let alone become someone to bring people and ideas together in editing and writing books about geochemical and extraterrestrial topics - nor do I know any so-called literati (indeed, what is that word?). A fateful phrase was once uttered though, "You can't do that". Unfriendly sentiments are to be discouraged (including those emanating from my cheeky nephew), but - and I hate to be predictable - in my case these can be relied upon to have an opposite effect. After all, it is always better to try and fail (then try again) than not to try at all. At the moment, and among other things, I have deviated from my previous norm to write stories of science for children. We have been locked-down during 2020, and I feel fortunate that reflecting and writing in quiet with the company of the cat has been a safe sanctuary.

Some people of science do in fact practice friendly non-technical writing from the time of their own childhood (who knew?!). Alas, my own youth was spent climbing trees, building dens in hedgerows, and then working a good number of parttime jobs, which left me little time for anything but walking the dog and playing basketball. Since then a considerable amount of time, focus, and intellectual weight-lifting has been centred on "serious scienc'ing". Much catching up to do in sci. comm! In diving into this type of writing I

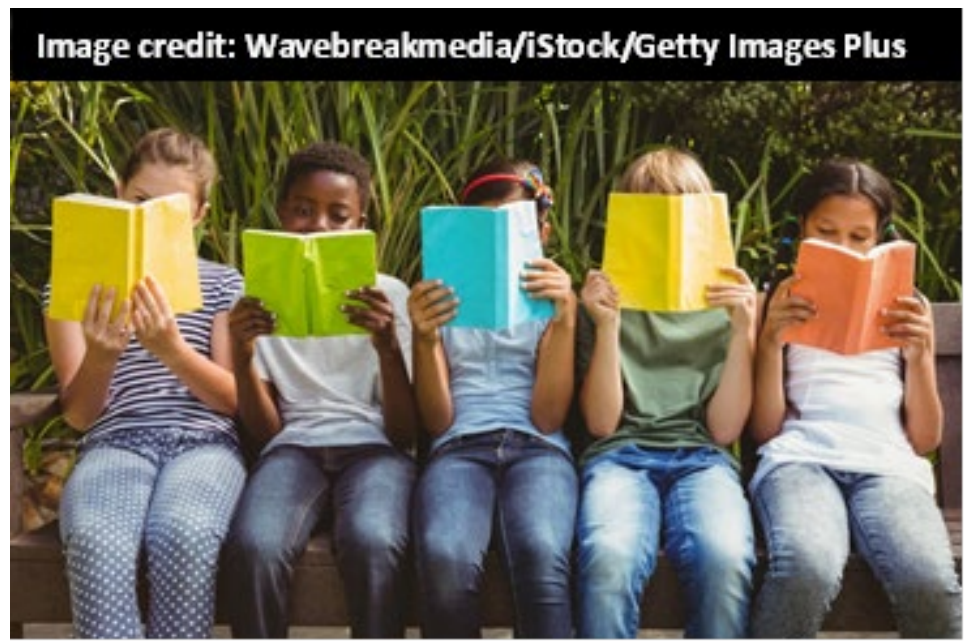


realised the (forgive the geo-pun) mountainous size of the task ahead. Storytelling of science requires characters and a dose of humour. A newly developed awareness drew my attention to the presently horrifying lack of diversity among the characters and protagonists of children's literature. For example, a recent survey of children's books by the Centre for Literacy in Primary Education (UK) revealed that less than $5 \%$ (!!) of protagonists are people of colour, and many other large studies report similarly low figures. Examinations of a few thousand books published in the US in 2017 have provided a maximum of $22 \%$ of characters representing ethnic minorities in child literature. This reality in publishing is in stark contrast to the fact that around $33 \%$ of school-aged child readers in any given country, much more globally, identify as people of colour themselves. These shocking factors underscore the need for us to provide a wider range of STEM-related role models in literature intended for formative ages. There is no clear trail of evidence for us so trained. Yet, clues suggest that it is no coincidence that a lack of storytelling and imagery representing a diversity of characters, including among historical or fictional scientists, for young readers ultimately results in a generation with preconceived notions about who scientists are, where they come from, and what scientists look like - perceptions unhelpful to inclusiveness. Are these the earliest of barriers thwarting the attainment of academic STEM communities that more closely reflect the populations that they serve? It is probably reasonable to postulate that these are significant contributing factors and we should not only be aware of this but - as with all obstacles to diversity, equity, and inclusion - must do something about it.

Many STEM academics around the world have blazed this trail in negotiating / making time to share their own zeal for science so as to shape the perceptions that young people have of STEM. For example, scientists who are parents may initially venture into storytelling for their own children before sharing such scientific tales and adventures more widely. Others may have published journalistic articles or stories in small magazines as 'hobbies' all of their lives, and before progressing to the not insignificant task of working with their own inner child in crafting full books for the entertainment of others. Yet more members of STEM work with children's authors and producers of science-based TV programs in an advisory capacity so as to aid and influence the content of children's literature and at-home family learning. Hence, these types of contributions are established and should be accepted by peers and / or managers as part of our careers. Given the shocking truth about the lack of diversity among characters represented in books for the youth I believe that we have a moral responsibility to raise this in all interactions and activities related to the art of writing creatively for children. Consequently, I have written this article while desk-bound under COVIDrestrictions (though applicable at all other times) to call for the good people of STEM to do more. The evidence of a pressing need for us to do things differently to ensure that we help every kind of person engage with and trust in STEM is undeniable. It is never too soon to start! Is writing for children also necessary to affect progressive long-term change in our higher institutes of learning and discovery? This is a theory hard to test, but I think it likely and hope that others agree. Give it a try, join in, so that we are all part of making a positive difference for the present and for the future. If it happens to work-out the community will never be the same again (in the best of ways), and additional outreach and enabling scholarship / access funds might even be raised along the way.

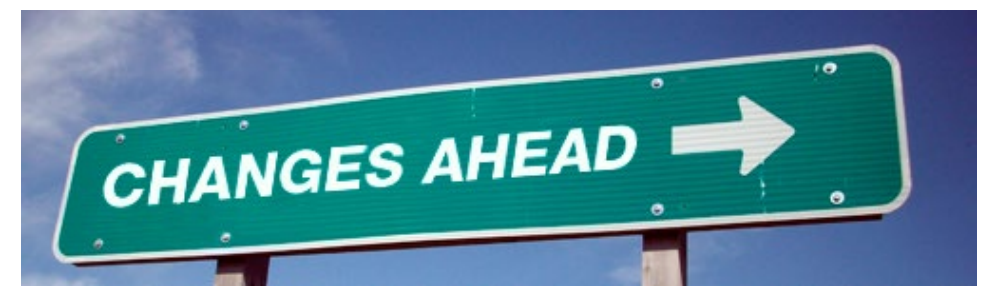

Seeking out a few affable science writers for friendly advice helps if newly venturing into such activities; Goldschmidt 2021 will provide a workshop on science communication as part of the early career program. 


\section{Bio}

Dr. Amy J. V. Riches' originally trained as a geologist and is now a dedicated and passionate planetary scientist, isotope geochemist and petrologist. She is revelling in intellectual freedom while freelance, is a Visiting Honorary Fellow at the University of Edinburgh, UK, and a SETI Affiliate Scientist, Carl Sagan Centre, USA. Amy's quantitative geochemical work is interdisciplinary in nature and has the goal of unmasking the

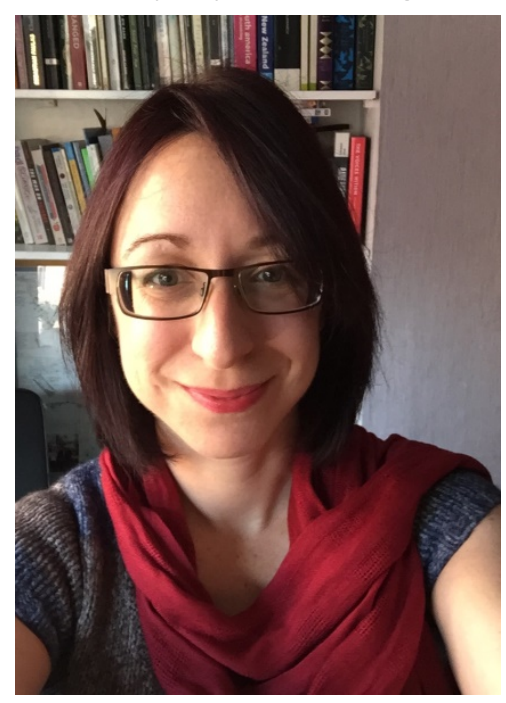
magmatic and interior compositions, origins, and evolutionary chapters of asteroids formed over 4.5 billion years ago, as well as improving understanding of Mars and the Earth-Moon system. The findings resulting from her interrogations of rocks from space, and the influence of such data on computational modelling of the physics of planetary construction and Solar System history, are needed to resolve long-standing controversies concerning the origin of Earth. This work serves to inform the search for habitable bodies elsewhere in the cosmos. To catch up on her work visit her website https://amyriches.org

As part of her wider contributions to the scientific community, Amy enjoys driving inclusive activities such as scientific meetings and edited volumes that advocate for and stimulate new multidisciplinary directions of study at international levels. Among these activities are forthcoming academic and popular science books (https://amyriches.org/books/) as well as the meeting "Forming and Exploring Habitable Worlds" intended for the summer of 2022 (https://amyriches.org/meeting-forming-and-exploring-habitable-worlds/), and the $5^{\text {th }}$ International Workshop on Highly Siderophile Element Geo- and Cosmochemistry Chaired by Thomas Meisel and planned to coincide with the opening of new facilities in September of 2022 in Leoben, Austria. In addition, Amy is among the 13 Founding Members of the European Association of Geochemistry's Diversity, Equity, and Inclusion Working Group led by Dr. Heather Buss, and whose ongoing work and team makeup will be more fully revealed to the EAG membership very soon. 